\title{
Urgent Surgical Management of Acute Lower Gastrointestinal Bleeding with Intraoperative Colonoscopy: A Case Report
}

\author{
Traore Amadou*, Konate Madiassa, Diarra Abdoulaye, Keita Soumaila, Kone Tani, \\ Karembe Boubacar, Amadou Issa, Sidibe Boubacar, Bah Amadou, Maiga Amadou, \\ Diakite Ibrahim, Dembele Bakary Tientigui, Traore Alhassane, Togo Adegne, \\ Kante Lassana, Samake Moussa, Mangane MoustaphaIssa, Diop Thierno Madane, \\ Diakite Adama, Diallo Gangaly
}

Gabriel Toure University Hospital, Bamako, Mali

Email:*amadoutra242@gmail.com

How to cite this paper: Amadou, T., Madiassa, K., Abdoulaye, D., Soumaila, K., Tani, K., Boubacar, K., Issa, A., Boubacar, S., Amadou, B., Amadou, M., Ibrahim, D., Tientigui, D.B., Alhassane, T., Adegne, T., Lassana, K., Moussa, S., MoustaphaIssa, M., Madane, D.T., Adama, D. and Gangaly, D. (2018) Paper Title. Surgical Science, 9, 300-305.

https://doi.org/10.4236/ss.2018.99036

Received: September 3, 2018

Accepted: September 14, 2018

Published: September 17, 2018

Copyright (c) 2018 by authors and Scientific Research Publishing Inc. This work is licensed under the Creative Commons Attribution International License (CC BY 4.0).

http://creativecommons.org/licenses/by/4.0/

\begin{abstract}
Lower gastrointestinal bleeding (LGIB) is defined as bleeding from a source distal to the Ligament of Treitz. The primary step is to identify severe hemorrhages that may be life-threatening for patients (10\% of cases of LGIB). The management depends on the severity of the bleeding, the diagnostic and therapeutic means of the patient's reception center. Patient with acute LGIB, in case of negativity of endoscopic and radiological examinations, can be a surgical indication with possibility of intraoperative colonoscopy. We report a case of urgent surgical management with intraoperative colonoscopy in a patient with severe acute LGIB.
\end{abstract}

\section{Keywords}

Lower Gastrointestinal Bleeding, Surgery, Gabriel Toure

\section{Introduction}

Lower gastrointestinal bleeding (LGIB) is defined as bleeding from a source distal to the Ligament of Treitz [1]. Its annual incidence is 20 cases/100,000 adults in France [2]. It represents about $20 \%$ of the digestive hemorrhages [3] [4]. The urgency is to know to recognize in time severe hemorrhages that may be life-threatening for patients (10\% of cases of LGIB) [4].

In spite of well led explorations, the diagnosis of the LGIB remains dubious in $10 \%$ of the cases. The recto-colic's causes account for $95 \%$ of the identified eti- 
ologies and are especially dominated by the diverticulosis [4].

If the LGIB is severe, in the event of negativity of the endoscopic and radiological examinations, surgery can be performed with possibility of intra-operative colonoscopy [1].

We report a case of urgent surgical management with intraoperative colonoscopy of a patient with severe acute low gastrointestinal hemorrhage.

We will discuss then the stages of diagnostic and therapeutic control.

\section{Case Presentation}

A 60 years old man was referred by the gastroenterology department for lower digestive tract hemorrhage with hemodynamic instability in April 2017.

The symptomatology began a year earlier with hematochezia made of bright red blood of low frequency, of little abundance; not worrying the patient at first. This rectal bleeding was accompanied by abdominal pain sitting at the level of the periumbilical region, a type of cramp of moderate intensity, intermittent radiating throughout the abdomen without triggering factors.

A week prior coming to the hospital, he had a massive hematochezia, occurring almost daily, made of bright red blood. This hematochezia was accompanied by abdominal pain, dizziness.

He consulted then at the gastroenterology department of Gabriel Toure University Hospital.

An emergency upper gastrointestinal endoscopy was normal. Completion of a colonoscopy the next day was made impossible by the abundance of hematochezia associated with the installation of hemodynamic instability. Based on the facts, the General Surgery Department was asked to discuss urgent surgical procedure.

In his history, the patient had high blood pressure and poorly monitored diabetes. He had never been operated. We did not find any particular family history or risk factors.

The general clinical examination revealed a WHO Performance Index rated III, a systolic/diastolic blood pressure of $80 / 60 \mathrm{mmHg}$, a heart rate of 120 beats per minute, a respiratory frequency of 30 cycles per minutes, a temperature of $37^{\circ} \mathrm{C}$, a weight of $87 \mathrm{~kg}$, a height of $1.9 \mathrm{~m}$ and a body mass index of $24.1 \mathrm{~kg} / \mathrm{m}^{2}$. He had conjunctival pallor; there was no edema of the lower limbs or the face.

On proctological examination, there was no hemorrhoid or anal fissure. At the rectal examination, we did not find an intraductal mass; the fingerstall was stained with bright red blood.

The rest of the examination was normal.

We have then made the following diagnostic hypotheses: hemorrhagic tumor of the colon and ulcerative colitis.

The biological assessment found a hemoglobin rate of $4 \mathrm{~g} / \mathrm{dl}$, the platelets rate of $171 \times 10^{3} / \mathrm{mm}^{3}$, glycemia rate of $22.20 \mathrm{mmol} / \mathrm{l}$.

Due to hemorrhagic shock, the patient was stabilized in intensive care after 
transfusion of 4 units of packed red blood cells.

After multidisciplinary consultation between anesthesiologist-resuscitator, gastroenterologist and surgeon, it was decided to perform an emergency surgical exploration assisted by an intraoperative colonoscopy to achieve hemostasis.

The surgical approach was a median laparotomy above and below umbilical. At first, the exploration did not find the source of bleeding. Intraoperative colonoscopy found two bleeding polyps in the right third of the transverse-colon (Figure 1) and diverticulosis of the colon.

A right hemicolectomy was performed, followed by an ileo-transverse colon anastomosis.

The postoperative management included monitoring of anemia which remained stable with a hemoglobin rate of $7.9 \mathrm{~g} / \mathrm{dl}$ on day 1 postoperatively then $8 \mathrm{~g} / \mathrm{dl}$ on day 3. The patient had an abdominal wall infection which was drained by the removal of some cutaneous sons. He was discharged after 18 days.

Histological examination of the specimen found a right colonic diverticulosis with inflammatory infiltrate and demonstrated that the two right colonic polyps were tubular adenomas.

A year after the procedure, the patient was well, without recurrence.

\section{Discussions}

Lower gastrointestinal bleeding accounts for $20 \%$ to $25 \%$ of all digestive tract hemorrhages [1] [4]. In North American studies, the annual incidence of LGIB is estimated to be between 21 and 27 per 100,000 population in adults [5] [6]. In France, it is estimated at 20 cases/100,000 adults. The incidence of LGIB is higher in men $(24.2 \%$ vs. $17.2 \% \mathrm{p}<0.001)$ and the elderly (increase of 200 times between 3rd and 9th decade of life) [6]. The average age of hospitalized LGIB patients ranges from 63 to 67 years [1] [2].

The classic clinical manifestation is the lower exteriorization of undigested blood (hematochezia) with or without a clot [3].

In contrast to upper gastrointestinal bleeding, the estimation of LGIB severity is not validated [1] [2] [3]. The evaluation of the gravity is however essential because it determines the outcomes. The severity of the hemorrhage, its occurrence within the hospital, its impact on the hemodynamic state, the need for resuscitation (transfusion, filling), the presence of comorbidities, the combination of an anticoagulant or anti-aggregating treatment and platelet rate are reported as a factor of severity [1] [2] [3].

The diagnostic and therapeutic procedures depend on the severity of the bleeding and means of the patient's reception center [1].

Management of severe acute LGIB begins with resuscitation, the goal of which is to stabilize the patient, transfuse if necessary, and correct hemostatic disturbances in order to perform the necessary safe explorations. It should be noted that LGIB are less severe than upper gastrointestinal hemorrhages with $80 \%$ spontaneous arrest and a mortality rate of $2 \%$ to $4 \%$ versus $6 \%$ to $13 \%$ [4] [7]. 


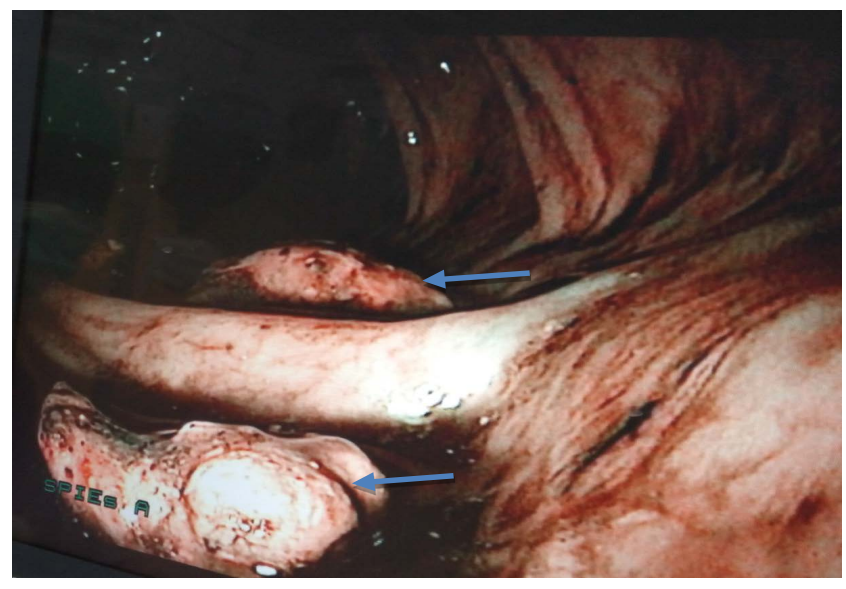

(a)

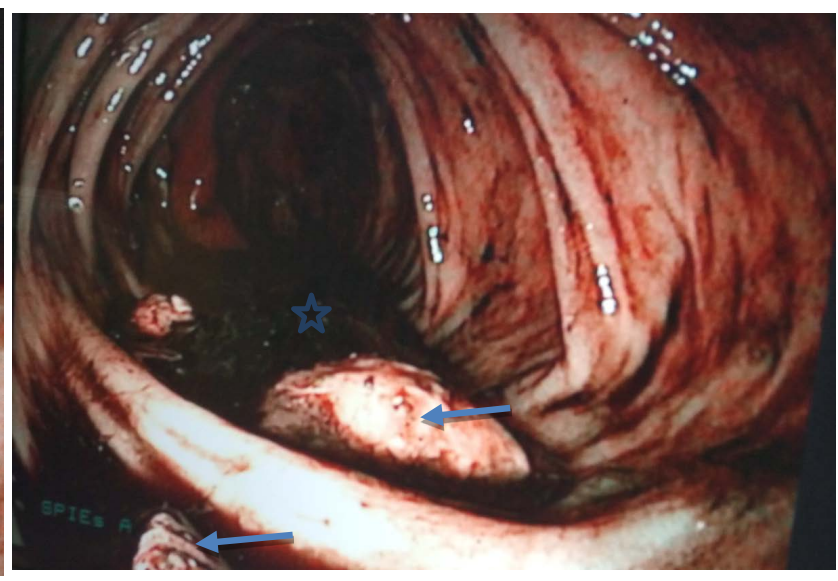

(b)

Figure 1. Pictures taken during intraoperative colonoscopy (a), (b) right third segment of the transverse colon. $\Rightarrow$ : polyps. At: blood.

In a case of severe acute LGIB, the trap is to miss an upper digestive hemorrhage ( $15 \%$ of the lower externalizations) [2]. The realization of upper gastrointestinal endoscopy had made it possible to exclude this diagnosis in our patient. In fact, this examination is recommended as first-line treatment for bleeding disorders with hemodynamic disorders [3].

The second step in the procedure is to propose a colonoscopy; his interest is both diagnostic and therapeutic. Even in case of emergency, it should always be performed on a previously prepared colon. Colonoscopy allows to explore the rectum, the colon and the last ileal segment. Emergency colonoscopy reveals the caecum in $55 \%$ to $70 \%$ of cases [8].

If preparation is not possible, an alternative to colonoscopy is CT angiography. It allows to locate an active hemorrhage and to guide a radio-embolization or a surgery [1].

If the endoscopic assessment is negative (gastrointestinal endoscopy and colonoscopy), the search for a small intestine cause of bleeding is recommended by the realization of an endoscopic video capsule (ECV) [1] [2].

The option of emergency surgery must be the subject of a multidisciplinary consultation between the resuscitator, gastroenterologist, radiologist and surgeon. It is indicated in 4 cases [1] [5]:

- Persistent hemodynamic instability despite optimal resuscitation;

- Persistent hemorrhage (>6 RBCs transfused) and no localization of bleeding despite colonoscopy, advanced enteroscopy and CT scan;

- Active bleeding from a segmental digestive lesion accessible to surgical hemostasis;

- The patient is a candidate for surgery without prohibitive comorbidity and with a reasonable life expectancy.

Emergency surgery was performed in $18 \%$ to $25 \%$ of LGIBs that required transfusion (grade B) [5]. Gayer and Col. in their study, they showed that mortality was lower if the bleeding was localized preoperatively, 3.3\% versus 9.4\% [9]. 
The advantage of previously locating the origin of the bleeding is to allow a suitable elective surgery that is opposed to an empirical surgery that is performed in the situation where the origin of the bleeding is not known. This empirical surgery is associated with high mortality. In fact, mortality was $10 \%$ to $33 \%$ in subtotal "blind" colectomy for bleeding of colonic origin and a re-bleeding rate of $0 \%$ to $8 \%[10]$.

To allow a suitable surgery in a situation where an urgent laparotomy has been performed without having localized the origin of the bleeding, a colonoscopy or an intraoperative gastrointestinal endoscopy is performed. The surgeon can perform an intraoperative colonic cleaning and guide the endoscopist [8] [11].

Roldan and col. reported 3 cases of severe LGIB with hemodynamic instability originating from small intestine that were managed by an urgent laparotomy and total intraoperative enteroscopy performed with a colonoscope [12].

For our patient, the intraoperative colonoscopy identified two sessile polyps of the right side of the transverse colon that were identified as the origin of the bleeding. It was decided to do a right Hemicolectomy in the event of a neoplastic nature of these polyps.

Gabriel and Col. reported two cases of acute LGIB with hemodynamic instability which were resolved in different ways. The first patient was successfully managed by angiography and embolization. But the second patient underwent surgery after the angiography performed being unable to locate the source of bleeding [13].

The histological results rather raised questions of the real origin of this hemorrhage due to the showing of diverticulosis of the right colon with inflammatory components next to the two polyps. These diverticula were not identified as the source of bleeding during the colonoscopy probably because of the difficult context of its realization.

However, in the literature, colonic diverticulosis is the leading cause of LGIB with a frequency of $26 \%$ to $38 \%$. Taking aspirin and NSAIDs have been identified as triggers. Age over 50 is a contributing factor. Polyps account for $4 \%$ to $14 \%$ of LGIB causes while cancers represent $8 \%$ to $12 \%$.

In all these cases, performing a right colectomy may be the cure for bleeding and its cause [1] [2] [3].

\section{Conclusion}

Even severe, LGIB rarely requires urgent surgical procedure. This is decided after a multidisciplinary consultation. The decision depends on the severity of the hemorrhage, its impact on the hemodynamic state and the limitation of less invasive means. Even in a state of emergency, it is essential to look for the source of the bleeding by an endoscopic assessment in first intention, in order to propose a suitable surgery.

\section{Consent}

We confirm that Consent has been obtained. 


\section{Conflicts of Interest}

The authors declare no conflicts of interest regarding the publication of this paper.

\section{References}

[1] Marion, Y., Lebreton, G., Le Pennec, V., Hourna, E., Viennot, S. and Alves, A. (2014) Prise en charge des hémorragies digestives basses [The Management of Lower Gastrointestinal Bleeding]. Journal de Chirurgie Viscérale, 151, 194-204. https://doi.org/10.1016/j.jchirv.2013.12.006

[2] Ah-Soune, P. and Barthet, M. (2015) Hémorragie Digestive Basse: Algorithme de Prise en Charge. Acta Endoscopica, 45, 321-324. https://doi.org/10.1007/s10190-015-0512-3

[3] Pioche, M. and Ponchon, T. (2017) Prise en Charge des Hémorragies Digestives Basses. EMC-Gastro-Enterologie, 12, 1-8.

[4] Artpurt, J.P., Lesur, G., Heresbach, D. and Soudan, D. (2010) Consensus en Endoscopie Digestive: Hémorragie Digestive Basse Aiguë. Acta Endocscopia, 40, 379-383. https://doi.org/10.1007/s10190-010-0110-3

[5] Farrell, J.J. and Friedman, L.S. (2005) Review Article: The Management of Lower Gastrointestinal Bleeding. Alimentary Pharmacology \& Therapeutics, 21, 1281-1298. https://doi.org/10.1111/j.1365-2036.2005.02485.x

[6] Longstreth, G.F. (1997) Epidemiology and Outcome of Patients Hospitalized with Acute Lower Gastrointestinal Haemorrahage: A Population-Based Study. The American Journal of Gastroenterology, 92, 419-424.

[7] Holster, I.L. and Kuipers, E.J. (2012) Management of Acute Nonvariceal Upper Gastrointestinal Bleeding: Current Policies and Future Perspectives. World Journal of Gastroenterology, 18, 1202-1207. https://doi.org/10.3748/wjg.v18.i11.1202

[8] Lhewa, D.Y. and Strate, L.L. (2012) Pros and Cons of Colonoscopy in Management of Acute Lower Gastrointestinal Bleeding. World Journal of Gastroenterology, 18, 185-190. https://doi.org/10.3748/wjg.v18.i11.1185

[9] Gayer, C., Chino, A., Lucas, C., Tokioka, S., Yamasaki, T., Edelman, D.A. and Sugawa, C. (2009) Acute Lower Gastrointestinal Bleeding in 1,112 Patients Admitted to an Urban Emergency Medical Center. Surgery, 146, 600-607. https://doi.org/10.1016/j.surg.2009.06.055

[10] Pilleul, F., Buc, E., Dupas, J.L., Boyer, J., Bruel, J.M. and Berdah, S. (2007) Prise en charge de l'hémorragie d'origine diverticulaire. Gastroentérologie Clinique et Biologique, 31, 47-52.

[11] Lee, J., Costantini, T.W. and Coimbra, R. (2009) Acute Lower Gi Bleeding for the Acute Care Surgeon: Current Diagnosis and Management. Scandinavian Journal of Surgery, 98, 135-142. https://doi.org/10.1177/145749690909800302

[12] Roldán, F.P., Carro, P.G., García, M.C., Yeste, J.P. and Villarín, A.L. (2009) Urgent Intraoperative Total Enteroscopy with Colonoscopy by Means of a Double Enterotomy in a Severe Lower Digestive Tract Haemorrhage. Cirugía Española, 86, 252-253. https://doi.org/10.1016/j.ciresp.2009.03.022

[13] Gabriel, N.A., Bogdan, P., Laurentiu, G., Bogdan, I.D., Bogdan, V.M. and Mircea, B. (2016) Highlighted Steps of the Management Algorithm in Acute Lower Gastrointestinal Bleeding-Case Reports and Literature Review. Chirurgia, 111, 74-79. 